\title{
Familial inheritance of TMD: a case report
}

Maria Genello ${ }^{1}$

${ }^{1}$ University of Pittsburgh, School of Dental Medicine

\section{Abstract}

The TMJ is a joint that can perform both hinge and sliding motions and whose proper functioning depends on the actions of the joint and joint capsule, the muscles of mastication, and the condition and action of the mandibular condyle. If any of these components deviate from their normal structure or function, then TMD can result. There have been many reported contributing factors to TMD development, many of which include environmental influences such as bruxism, trauma, and other oral habits. However, a genetic component can also play a role. This paper reports a case of TMD occurring within three consecutive generations of a family and explores the possibility of this disorder exhibiting familial inheritance. Members of the family affected all displayed manifestations of joint laxity in other areas of the body, migraines, pain, and had smaller craniofacial dimensions with a narrower mandible. These factors were found to have a genetic influence and these genes can also be tied to TMD, thus supporting the argument that the cases of TMD seen in this family are in fact due to inheritance. If TMD can be shown to have a genetic component and be inherited, then dental practitioners would be able to identify high risk patients and help to modify environmental factors early on in order to help prevent the onset of TMD in those individuals.
Citation: Genello, M. (2017) Familial inheritance of TMD: a case report. Dentistry 3000. 1:a001 doi:10.5195/d3000.2017.75

Received: May 15, 2017

Accepted: May 26, 2017

Published: June 16, 2017

Copyright: (C2017 Genello, M. This is an open access article licensed under a Creative Commons Attribution Work 4.0 United States License.

Email: mkg35@pitt.edu

\section{Introduction}

The TMJ is a unique bilateral joint that connects the mandible to the temporal bone [1]. It performs both hinge and sliding motions which allows a person to talk, chew, yawn, and open the mouth. Some of the component parts of the TMJ include the mandibular condyle, the glenoid fossa of the temporal bone, the articular eminence which the condyle slides down, the articulating disc between the condyle and temporal bone, and the joint capsule. During function, the condyle glides forward from the fossa and down the articular eminence. The disc remains between the condyle and these other elements in order to act as a shock absorber [1]. A disorder of the TMJ, termed TMD, can affect one or both of the joints. Manifestations of TMD include, but are not limited to, pain in the face, limited movement and stiffness of the jaw, locking of the jaw, and popping or clicking of the joint upon opening [1]. The cause of TMD has been attributed to many different factors, a large portion being environmental. These include trauma, bruxism, and other oral habits. A genetic component is being explored, and many different genes have been proposed as possible candidates.

\section{Case Report}

Three generations of a family from northeastern Pennsylvania were analyzed for TMD. The disorder appeared in each generation, and appeared to affect predominately the females in the family. In generation III, two males appear to be affected. All of those that report having manifestations of TMD have the common feature of clicking and popping upon jaw opening. In generation one, (will refer to as "female $A$ "), the affected female $A$ reports having symptoms of popping and clicking, along with mild hip and knee joint laxity. She also reports mild intermittent jaw pain and headaches.

In generation two, three females (females B, C, and D respectively) are affected out of four, and $50 \%$ of the offspring are affected. The affected females all report jaw clicking and popping upon opening, with female $B$ reporting mild intermittent headaches and occasional, mild jaw pain. Female $C$ reports clicking and popping as well. She suffers from migraine headaches that occur every few months. She has laxity in her left knee joint, and reports frequent jaw pain that oftentimes is severe. Her jaw mobility frequently becomes restricted, causing her to have limited opening. Female $D$ reports clicking and popping, along with mild jaw dislocation when she opens wider than her normal functional range (for speaking, chewing, etc). She (c) BY

ULLS D-Sorle
New articles in this journal are licensed under a Creative Commons Attribution 4.0 United States License.

This journal is published by the University Library System, University of Pittsburgh as part of its D-Scribe Digital Publishing Program and is cosponored by the University of Pittsburgh Press. 
exhibits mild intermittent jaw pain. This same female $D$ also reports laxity in her intervertebral discs, and has suffered two "slipped" discs. She also reports more severe intermittent headaches.

In generation three, four members are affected, two of which are males. Male A is the offspring of female $B$, and presents with only occasional mild clicking and popping upon opening. Male $B$ is the offspring of female $C$, and reports only occasional clicking and popping that is mild. Female $E$, who is the offspring of female $C$, presents with clicking and popping when opening the jaw, along with severe left shoulder joint laxity. She also reports occasional moderate headaches and mild, intermittent jaw pain. Female $F$, who is the offspring of female $D$, reports clicking and popping, along with occasional migraine headaches and intermittent, more moderate jaw pain. When opening past functional limits, the jaw often becomes dislocated. She also exhibits laxity to her intervertebral discs and has suffered a herniation, along with laxity to her hip joint.

Of the affected individuals, all but male $B$ have smaller vertical and horizontal craniofacial measurements, with a narrow mandible. Male $B$ exhibits a wider, less constricted mandible.

\section{Discussion}

TMD proves to be a disorder of variable expression and has many different manifestations. Many cases of TMD have a strong

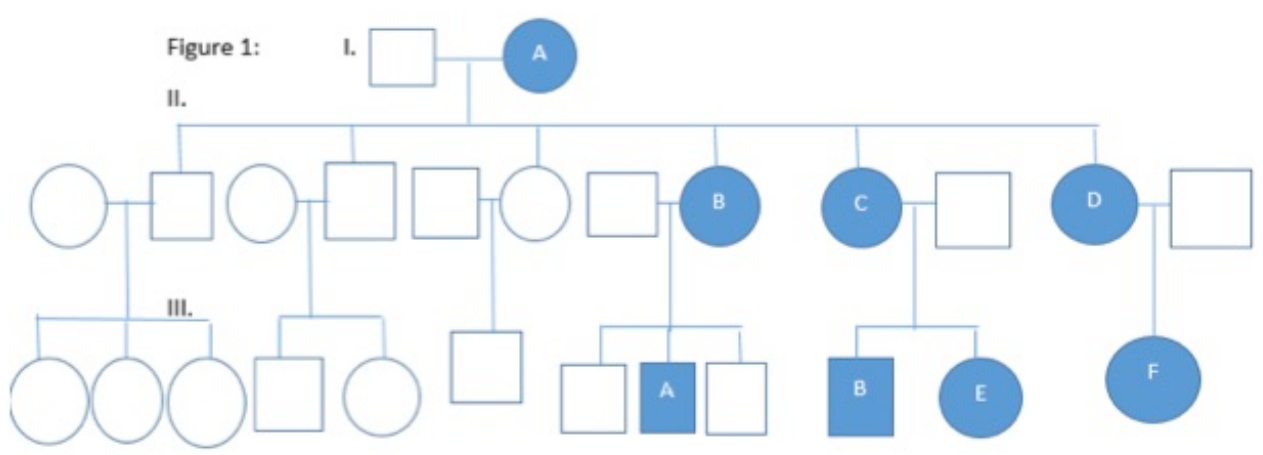

Figure 1. Pedigree of the affected family. Blue color indicates those that have any symptoms pertaining to TMD, whether they be mild, moderate, or severe. Letters are used as identifiers when discussing the case.

environmental component, however, genetic elements to the disease are being studied and suggested. The TMD evident in this family appears to have a genetic influence. When you look at figure 1, one sees how TMD appears to be present in all three generations. Mainly females are affected, with the ratio being $6: 2$ females to males. This is expected, since it is found that females, especially those of child bearing age, are more susceptible to developing TMD and have more severe symptoms due to hormonal differences [2].

If we were to propose the most common mode of inheritance for TMD, it would most likely be polygenic inheritance with environmental influences. Since the TMJ function depends on a multitude of factors such as masticatory musculature, articular disc formation and health, integrity of the joint capsule, shape and angle of the condyle, mandibular size and development, shape of the glenoid fossa, integrity of the cartilage, etc., any variation in these or other structures that influence its function can result in a deviation from normal and lead to TMD.
Since these traits are all controlled by many and different genes working together in order to achieve proper development and function, it would be safe to say that any TMD with a strong genetic component would be polygenic, meaning there would be variations in multiple genes that influence the structures relevant to the TMJ. This can also account for the variable expression of TMD. Since many different genes can have variations that lead to TMD, not just a single gene, and different environmental factors, and not just a single environmental factor, can aid in the development of $T M D$, then the expression seen in the population of TMD would be variable.

In this family's case, therefore, the inheritance is most likely polygenic. Those affected appear to have a myriad of symptoms that deal with different aspects of the TMJ, and the expression of TMD within the family isn't completely uniform, but is rather somewhat variable, leading us to believe in the polygenic theory. Now, if we are speaking in terms of autosomal dominance and recessiveness, or X-linked domi 


\begin{tabular}{|c|c|c|c|c|c|c|}
\hline $\begin{array}{l}\text { Affected } \\
\text { Member }\end{array}$ & $M / F$ & Clicking/Popping & $\begin{array}{l}\text { Headaches } \\
\text { (mild, } \\
\text { mod., } \\
\text { severe) }\end{array}$ & $\begin{array}{l}\text { Systemic joint } \\
\text { laxity/location }\end{array}$ & $\begin{array}{l}\text { Pain } \\
\text { (mild, } \\
\text { mod., } \\
\text { severe) }\end{array}$ & $\begin{array}{l}\text { Movement } \\
\text { restrictions }\end{array}$ \\
\hline $\bar{A}$ & Female & Yes & Yes; mild & $\begin{array}{l}\text { Yes; hip and } \\
\text { knee }\end{array}$ & Yes; mild & No \\
\hline$B$ & Female & Yes & Yes; mild & No & Yes; mild & No \\
\hline C & Female & Yes & $\begin{array}{l}\text { Yes; se- } \\
\text { vere }\end{array}$ & Yes; knee & $\begin{array}{l}\text { Yes; } \\
\text { severe }\end{array}$ & Yes \\
\hline $\mathrm{D}$ & Female & Yes & $\begin{array}{l}\text { Yes; se- } \\
\text { vere }\end{array}$ & Yes; IV discs & Yes; mild & yes \\
\hline$A$ & Male & $\begin{array}{l}\text { Yes; mild and } \\
\text { occasional }\end{array}$ & No & No & No & No \\
\hline$B$ & Male & $\begin{array}{l}\text { Yes; mild and } \\
\text { occasional }\end{array}$ & No & No & No & No \\
\hline $\bar{E}$ & Female & Yes & $\begin{array}{l}\text { Yes; mod- } \\
\text { erate }\end{array}$ & Yes; shoulder & Yes; mild & No \\
\hline$F$ & Female & Yes & $\begin{array}{l}\text { Yes; se- } \\
\text { vere }\end{array}$ & $\begin{array}{l}\text { Yes; hip and } \\
\text { IV discs }\end{array}$ & $\begin{array}{l}\text { Yes; } \\
\text { moderate }\end{array}$ & Yes \\
\hline
\end{tabular}

nance and recessiveness, it is most likely an autosomal dominant inheritance form. It appears in every generation, ruling out a recessive inheritance pattern, and it affects both males and females, with the females at a higher instance, leading one to believe that it is not X-linked.

Now that we have established that it is likely polygenic with an autosomal dominant inheritance pattern, we must make the case that it is in fact genetic variations that exist in this family that are causing these clinical manifestations of TMD, and these variations are being passed from one generation to the next. The genetics behind pain perception and chronic pain have become an area of interest with regards to TMD. A few candidate genes have been highlighted, which includes the serotonin 5-HT transporter gene and the COMT gene, whose activity is inversely correlated to pain sensitivity and the development of TMD. It was found that nearly a third of new TMD cases reported could be attributed to having a variation in the COMT gene [2]. In our subjects here, we saw that pain was a common feature in all three generations, ranging from mild to severe. It is important to note that those with more manifestations of the disorder, such as female $\mathrm{C}$ and female $F$, experienced more severe pain than the others that were affected, indicating a possible correlation between pain sensitivity and TMD severity.

Another important finding in this family and that deals with chronic pain was the presence of headaches. Numerous studies have been done on the association of certain genes and headaches. Through these studies, it was found that some of these genes not only predisposed a person to headaches and migraines, but to TMD as well. COMT was again part of these studies. COMT genetic variations were involved with both migraines and TMD. DRD4 was another gene that is involved with neurotransmission and that was associated with both the presence of headaches and the manifestation of TMD [3]. Another gene of interest was the ESR1 gene, which variations lead to the presence of chronic headaches and was also associated with TMD manifestations. Studies show that up to $50 \%$ of migraine and chronic headache etiology was due to genetic variants [3]. Headaches were present in all female cases in this family, and ranged from mild to severe. The presence of this shared trait in all of the affected female individuals could be due to genetic variation, specifically a gene that not only deals with the presence of headaches, but can also be associated with TMD (like COMT, DRD4, and ESR1), which would explain the concurrent expression of both TMD and chronic headaches in all of the affected female individuals. 
A trait that many of the affected individuals of this family shared was that of systemic joint laxity. Joints affected ranged from the knee and hip joints, to the shoulder joint and intervertebral discs. Systemic laxity of joints has a genetic component. There are certain genes that control the amount and type of collagen produced in the body. Skin biopsies of patients with joint laxity found them to have less overall collagen and a higher ratio of type III collagen to type III+I [4]. Those with systemic joint laxity and hypermobility had a higher incidence of TMD. This is due to the fact that if the joints in the body are more mobile and lax, then the capsule of the TMJ would also be lax, making the disc vulnerable to displacement and the condyle prone to hypermobility [5]. So, a genetic component can be involved in the joint laxity and subsequent TMD seen in each generation of this family.

Another important feature to note in this particular family was the smaller craniofacial dimensions and narrower mandible. All of the affected individuals but one have a narrower and smaller face. A high score on the Helkimo's Clinical Dysfunction Index, which is a tool used to assess the presence and severity of TMD, was found in patients with smaller vertical and horizontal facial dimensions [6]. Facial development and size is under strict genetic control. Any variation in these developmental genes can cause variations in the size and shape of the craniofacial bones and their rela- tionship to each other, which can lead to a TMD. The variations in the gene pool of this family for the size and width of the craniofacial bones could be a factor in their shared TMD.

\section{Conclusion}

The genetic component of TMD, while not as extensively explored as the environmental aspect, presents with some compelling support. Many candidate genes have been identified to be associated with the development of TMD. The family involved in this case report saw TMD reported in three consecutive generations. There was some variation in the expression of the disorder, but most of those affected had clicking and popping of the joint, along with systemic joint laxity, headaches, and pain. Smaller craniofacial dimensions were also a common feature seen. All of these features have a genetic component that can also be tied to TMD. Therefore, the conclusion is that the TMD seen in three consecutive generations of this family is the result of genetic variation and is inherited. Furthermore, by demonstrating a genetic basis for many cases of TMD, gene mapping can be done in those families or in individuals thought to have a higher susceptibility for TMD, and thus analyze the genes involved and the risk of either developing a TMD or the risk of offspring acquiring TMD. If a dental practitioner is made aware of a patient's risk of TMD, then steps can be taken early on to modify said patient's environmental factors (such as reducing bruxism, clenching of teeth, etc.) in an effort to try to prevent or delay the onset of TMD.

\section{References}

1. TMJ disorders. National Institute of Dental and Craniofacial Research. [cited March 4, 2017] Available from https://www.nidcr.nih.gov/oralhe alth/topics/tmj/tmjdisorders.htm\# symptoms.

2. The many faces of the genetics contribution to temporomandibular joint disorder. Oakley M, Vieira AR. Orthod Craniofac Res. 2008 Aug;11(3):125-135. PMID: 18713149.

3. Genetic predictors of human chronic pain conditions. ZorinaLichtenwalter K, Meloto CB, Khoury S, Diatchenko L. Neuroscience. 2016 Dec;338:36-62. PMID: 27143481.

4. Temporomandibular joint dysfunction and systemic joint laxity. Westling L. Swedish Dental Journal Supplement. 1992;81:1-79. PMID: 1621231

5. The role of systemic hypermobility and condylar hypermobility in temporomandibular joint dysfunction. Kavuncu, V., Sahin, S., Kamanli, A. et al. Rheumatol Int. 2006 Jan;26(3): 257-60. PMID: 15988598

6. Temporomandibular disorders in relation to craniofacial dimensions, head posture and bite force in children selected for orthodontic treatment. Liselotte Sonnesen, Merete Bakke, Beni Solow. Eur J 
Vol 5, No 1 (2017) Dol 10.5195/d3000.2017.75

Orthod. 2001 Apr;23(2): 179-192.

PMID:11398555 